\title{
Propuesta de diseño de un sistema de torres de detección de incendios forestales: aplicación a la región Metropolitana de Chile central
}

\author{
System design of wildfire detection towers
}

\author{
Gonzalo Tapia $^{\text {a* }}$, Miguel Castillo ${ }^{b}$ \\ *Autor de correspondencia: a Corporación Nacional Forestal (CONAF), Paseo Bulnes 285, Santiago, Chile, gonzalo.tapia@conaf.cl \\ ${ }^{\mathrm{b}}$ Universidad de Chile, Laboratorio de Incendios Forestales, Santiago, Chile.
}

\begin{abstract}
SUMMARY
A location design of wildfire detection towers for Region Metropolitana is proposed. For that objective, critical zones of occurrence are determined based on the historical records of fires and afterwards, a boundary of quadrants --based on the net used by Corporacion Nacional Forestal (CONAF) for the record of fires-- is designed. Later the potential problems of occurrence and spread of wildfires are spatially and temporarily assessed by means of the determination of "protection priorities" in the study area. Subsequently, in order to minimize the quantity of optional locations for the emplacement of towers, the identification of prominent points of relief (geographical landmarks) present in the area is suggested. This is achieved through a topographic index that evaluates the altitude of every point in relation to his neighboring zones. Later, a combinatorial automated model is developed in GIS; this model evaluates the set of possibilities and allows determining the minimal quantity of towers capable of covering at least $75 \%$ of the surface in the first priority of protection to the interior of each quadrant. Results indicate that Region Metropolitana contains 16 critical zones of occurrence. On the other hand, the proposed system needs eight points of observation to cover in average $79.3 \%$ of the surface of the first priority contained by the quadrants and $46.8 \%$ in areas of equal qualification at regional level. The flexibility of the proposed model, of being repeatable in different zones and scales, stands out; allowing besides the incorporation of diverse restrictions before its evaluation.
\end{abstract}

Key words: wildfire detection, protection priorities, geographic information systems (GIS).

\section{RESUMEN}

Se propone un diseño de localización de torres para detección de incendios forestales, tomando como área de prueba la región Metropolitana de Chile Central, en Sudamérica. Como primer paso se efectuó la delimitación de zonas críticas de ocurrencia a partir de los registros históricos de incendios y una posterior delimitación de cuadrantes basados en la grilla empleada por la Corporación Nacional Forestal (CONAF) de Chile para el registro de incendios. Posteriormente, se evaluó espacial y temporalmente los problemas potenciales de ocurrencia y propagación de incendios forestales mediante la determinación de "prioridades de protección" en el área de estudio. Con el fin de minimizar la cantidad de localizaciones opcionales para el emplazamiento de torres, se desarrolló un modelo combinatorio automatizado en SIG que evalúa el conjunto de posibilidades y permite determinar la cantidad mínima de torres capaz de cubrir al menos un $75 \%$ de la superficie, en primera prioridad, de protección al interior de cada cuadrante. Los resultados indican que la región Metropolitana de Chile contiene 16 zonas críticas de ocurrencia. Por otro lado, el sistema propuesto requiere de ocho puntos de observación para cubrir en promedio, un 79,3\% de la superficie de primera prioridad contenida por los cuadrantes y un 46,8 $\%$ en áreas de igual calificación a nivel regional. Se destaca la flexibilidad del modelo propuesto al ser replicable en diferentes zonas y escalas, permitiendo además de la incorporación de diversas restricciones previas a su evaluación.

Palabras clave: detección de incendios forestales, prioridades de protección, sistemas de información geográfica.

\section{INTRODUCCIÓN}

En Chile la temporada de incendios forestales comienza entre los meses de octubre-noviembre, cuando las condiciones climáticas favorecen el inicio y la propagación del fuego. Se destacan los meses de enero y febrero como los más conflictivos, observándose un descenso de la ocurrencia a partir del mes de abril, principalmente, por efecto de la estacionalidad. Durante la temporada 2013-2014 (datos oficiales de la Corporación Nacional Forestal obte- nidos al 20 de febrero de 2014) se registraron 249 incendios forestales en la región Metropolitana, con una superficie total afectada de 21.189 ha. Esta cifra está, por lejos, sobre el promedio histórico de área quemada de los últimos 10 años (826 ha por año). El descuido de conductores y transportistas, la imprudencia de las personas al manipular fuego y la mayor accesibilidad a nuevas zonas producto de la actividad turística, se cuentan como las principales causas de incendios en la región (CONAF 2003, 2006, 2014). 
La detección temprana de incendios se constituye en una actividad fundamental en manejo del fuego para aminorar oportunamente los efectos de la ocurrencia. Corresponde a la acción dirigida a vigilar una zona bajo protección, con el propósito de descubrir y localizar los incendios forestales que puedan haberse iniciado (Brown y Davis 1973). Esta acción puede clasificarse en no programada y programada. La primera incluye todos los medios informales que son capaces de registrar y procesar los avisos de descubrimiento y localización de focos (Davis et al. 1959). La segunda categoría contempla los medios que se han dispuesto organizadamente para la detección de incendios forestales, entre los cuales cabe mencionar los siguientes sistemas: terrestre fijo, terrestre móvil y aéreo (Julio 2005). El sistema de detección terrestre fija se basa en la utilización de torres de observación, construidas principalmente de madera o metal, en cuya parte superior se ubica un vigía o torrero. Su altura puede variar entre 5 y 30 metros; a la vez que su radio de visibilidad está determinado por las condiciones atmosféricas y topográficas del emplazamiento, variando entre 5 y $30 \mathrm{~km}$; siendo $20 \mathrm{~km}$ el estándar utilizado en Chile (Julio 2005). En la región Metropolitana de Chile existen tres torres de observación: Chicureo, Los Ratones y Antilén; ubicados en las comunas de Colina, Pirque y Recoleta, respectivamente. Según los propios encargados del Programa de Manejo del Fuego en la región, su localización responde más a oportunidades de construcción que a estudios técnicos que hayan determinado su ubicación. Asimismo la visibilidad de las torres existentes comprende principalmente zonas urbanas. Por otro lado, la región cuenta con una red de patrullaje terrestre diseñado para otros fines, sumado a un sistema de detección aérea discontinua y poco frecuente.

Antes de definir un programa de detección se debe evaluar la distribución espacial de los problemas potenciales de ocurrencia y propagación de incendios forestales; para ello, puede utilizarse la propuesta desarrollada por Julio (1992) conocida como el "método de determinación de prioridades de protección", que califica los diferentes sectores de una zona o región con el fin de identificar la demanda de recursos y facilitar la asignación de medios conforme a su importancia.

A partir del análisis previo y debido a la imposibilidad física y económica de establecer una cobertura de protección para toda el área de estudio, resulta de utilidad determinar las zonas críticas de ocurrencia de incendios. Estas no siempre pueden obtenerse mediante un simple método estadístico, por lo que debe utilizarse un análisis de vecindad con el fin de suavizar diferencias muy altas en la magnitud de los valores de ocurrencia entre las unidades vecinas. El proceso recién descrito puede ser ejecutado mediante sistemas de información geográfica (SIG) a través de un filtro espacial de medias, el cual asigna a cada unidad de análisis un valor calculado con el promedio aritmético de las ocho unidades vecinas. Para obtener mejores resultados, el proceso debe iterarse hasta lograr una estabilización en la desviación estándar de las imágenes (Castillo y Pedernera 1999, Castillo et al. 2004).

Una vez determinadas las zonas de interés, debe seleccionarse un conjunto de puntos opcionales de observación. Según lo indicado por Pedernera (1995) esto puede abordarse mediante dos opciones: la primera y más utilizada, se basa en el conocimiento y la experiencia de encargados de programas de manejo del fuego, quienes determinan una serie de puntos aptos como opciones de localización. La segunda, consiste en realizar un análisis SIG punto a punto que consigne los criterios de localización. Este sin embargo, representa un proceso demasiado complejo, debido al tamaño de la matriz de datos a analizar.

Para sortear dicho obstáculo, diversos autores han planteado diferentes métodos con el fin de optimizar el proceso de selección, ya sea reduciendo la cantidad de operaciones mediante el cálculo de índices de visibilidad (Franklin y Vogt 2004) o utilizando sólo hitos geográficos como puntos candidatos (Chandler et al. 1986, Rana y Morley 2002, Kim et al. 2004). Esta última propuesta se basa en la correlación existente entre el grado de visibilidad y la posición topográfica del candidato seleccionado. Hitos geográficos tienden a poseer un mayor porcentaje de visibilidad (Kim et al. 2004). De esta forma, seleccionando los puntos críticos del relieve, ubicados en el área de estudio, se puede reducir la cantidad de opciones y, por ende, disminuir los tiempos y procesos necesarios para los análisis de visibilidad. Mediante SIG estos puntos pueden obtenerse utilizando el índice topográfico de posición (TPI) desarrollado por Weiss (2001). Este calcula la relación entre la altitud de cada píxel con sus unidades vecinas, considerando el promedio aritmético y la desviación estándar. Celdas con valores positivos altos en el índice son identificados como cimas, mientras que la situación contraria califica a estas como fondos de valles. No obstante terrenos extensos sin variación de altura y por ende, carentes de hitos geográficos, no permiten la identificación de puntos destacables.

Se han desarrollado diversos métodos para definir la localización óptima de unidades en temas referentes a incendios forestales. Pedernera (1995) propuso un modelo de programación entera binaria, con el fin de determinar la cantidad mínima de torres de detección para vigilar el patrimonio de una empresa forestal, sujeto a restricciones de cobertura en las unidades de análisis (predios) y una superficie mínima aceptable cubierta por el sistema, en prioridades altas de protección. Posteriormente Pedernera (2004) publicó un estudio en el que intentaba conocer la localización óptima de brigadas de combate de incendios forestales a través de un modelo aditivo. En dicho trabajo seleccionaba en primer lugar, la unidad con mayor visibilidad para luego ir agregando una a una, la brigada con mayor aporte de cobertura al sistema hasta que la incorporación de un nuevo componente no produjese un aporte marginal significativo (Pedernera 2004). El primer método descrito hace imposible la incorporación como restricción de los traslapes en la visibilidad de las unidades, pues en un principio 
se desconocen las combinaciones que evaluará el modelo $\mathrm{y}$, por ende, sus traslapes. El segundo procedimiento, a pesar de incluir los traslapes de visibilidad, queda sujeto a la selección de la primera unidad, impidiendo reemplazar algún componente a medida que se evalúa el modelo. Los resultados obtenidos con este último representan una solución adecuada, pero no necesariamente la óptima. Por otro lado, Echeverría (1995) desarrolló un modelo combinatorio para la localización de brigadas de combate terrestre de incendios forestales. Este método, utilizado posteriormente por Aliste (2006) para la evaluación de helipuertos, se basaba en la exploración de todos los resultados posibles para cada nivel disponible (número de brigadas $=1,2, \ldots$, 16), con el fin de maximizar el área cubierta, considerando un total de 16 localizaciones para cada zona delimitada. El estudio incluía un área ubicada al norte del río Maule, y otra al sur de este mismo. Este método permite, además de considerar los traslapes, restringir la selección de puntos candidatos, sobre la base de una superficie mínima aceptable de acuerdo al análisis de prioridades de protección.

Considerando los antecedentes presentados anteriormente, esta investigación tiene por objetivo general diseñar un sistema de torres de detección de incendios forestales para la región Metropolitana de Chile central, que cumpla con la calificación y priorización espacial de las torres para la detección de incendios. La metodología aquí propuesta es ciertamente aplicable a otras regiones e incluso países, en la medida que se cuenten con los datos básicos para el desarrollo del sistema.

El área de estudio corresponde a un clima mediterráneo con una marcada estacionalidad entre invierno y verano, con una vegetación de bosque y matorral abierto, dominado principalmente por especies del antiguo bosque mediterráneo que dominó estas latitudes hace más de 10 mil años.

\section{MÉTODOS}

Delimitación de zonas críticas y cuadrantes de ocurrencia. La Corporación Nacional Forestal (CONAF), a través de su división de estadísticas, genera todos los años las cifras de ocurrencia y causas de incendios forestales, las cuales se encuentran disponibles en internet para acceso público. Sobre la base de esta información se creó una cobertura vectorial de puntos (figura 1A) la cual fue transformada a formato raster que representó la frecuencia de incendios ubicados dentro de una misma unidad $\left(1 \mathrm{~km}^{2}\right)$, en el período 2000-2014 (figura 1B). Para delimitar la zona crítica de ocurrencia se aplicó de forma sucesiva un filtro espacial

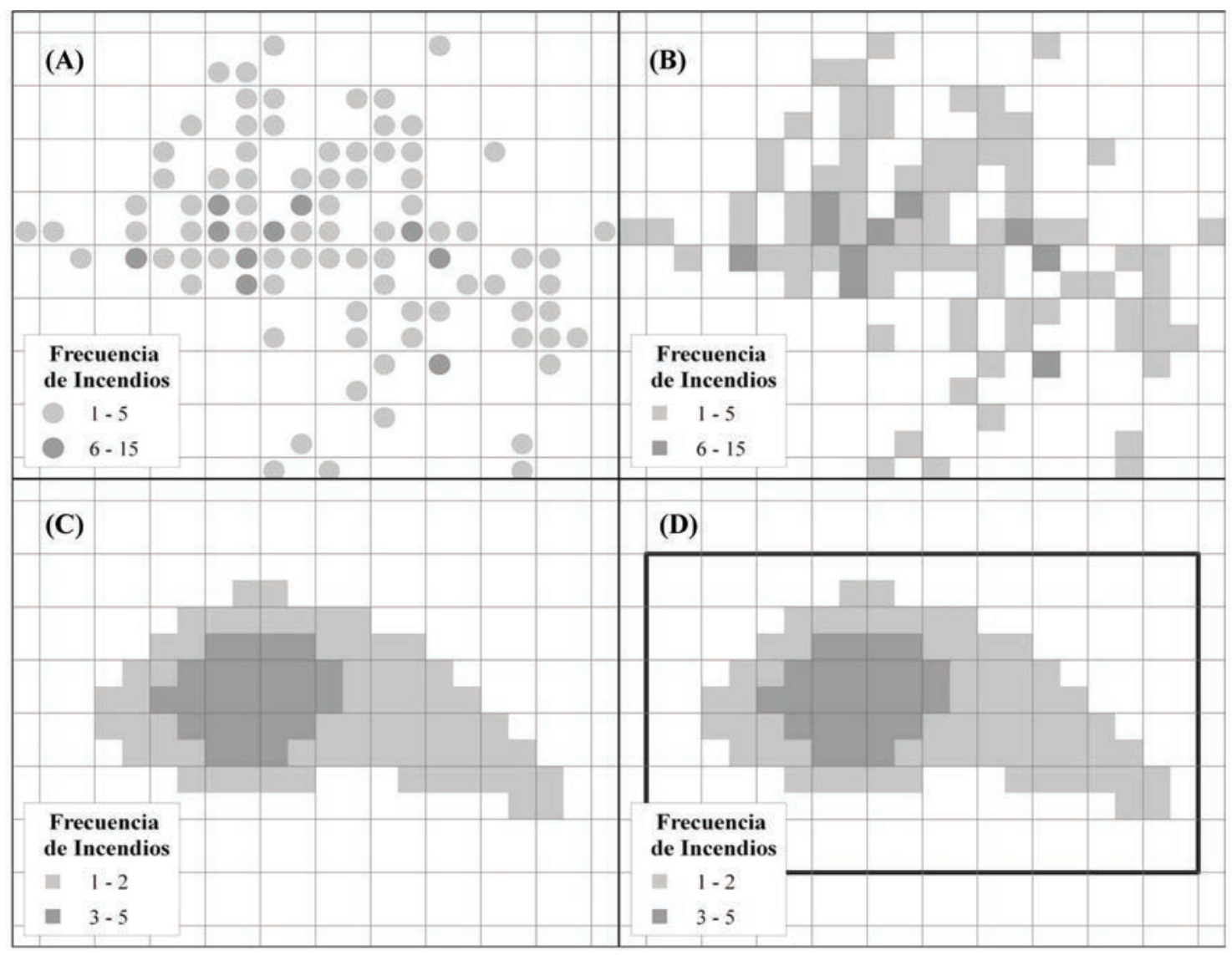

Figura 1. Etapas para la delimitación de cuadrantes de ocurrencia crítica de incendios.

Steps to delimit critical quadrants of fire occurrence. 
de medias en análisis raster, utilizando un área de $3 \times 3$ píxeles hasta obtener una estabilización en la desviación estándar de las imágenes (figura 1C). Se crearon a continuación cuadrantes seleccionando todas aquellas cuadrículas (píxeles) donde la ocurrencia fuese igual o superior a un incendio (figura 1D).

Determinación de prioridades de protección. Para determinar las prioridades de protección en la región Metropolitana se utilizaron los puntajes propuestos por Lathrop (2004) quien obtuvo estos a partir de la aplicación del Método Delphi a una serie de profesionales relacionados con el tema de incendios forestales. Este estudio de prioridades contempló el desarrollo de tres ejes de análisis: el riesgo, peligro y daño potencial de los problemas territoriales que se presentan normalmente en la región. Como complemento al estudio señalado se añadieron las estadísticas de incendios para el período 2000-2014, una nueva cobertura de caminos y la información agroclimática no incluida anteriormente.

Selección de puntos opcionales de observación. Como primer paso para la selección de puntos opcionales de observación, se creó un modelo digital de terreno (MDT) de la región Metropolitana, a partir de curvas de nivel separadas a intervalos de $25 \mathrm{~m}$ (figura 2A). A continuación se clasificó topográficamente el MDT con el fin de identificar los hitos geográficos de relieve presentes en el área (figura 2B).
Una vez definidas las zonas calificadas como hitos, se calculó el centroide de cada polígono generado, designando así un único punto representativo en cada zona (figura 2C). Examinando luego la cobertura generada se eliminaron todos aquellos puntos que no correspondían a la máxima altitud en su respectiva celdilla o pixel (figura 2D).

Como etapa previa al cálculo de las visibilidades, se examinó el radio de visibilidad adecuado para la condición topográfica de cada punto. Se probaron radios a partir de $5 \mathrm{~km}$, en intervalos de igual distancia, hasta un máximo de $20 \mathrm{~km}$. Se designó luego, para cada punto opcional, aquel radio que entregaba mayor visibilidad en zonas de primera prioridad de protección. Posteriormente se determinaron las zonas visibles en primera prioridad de protección de cada uno de los puntos, considerando una altura del observador de $20 \mathrm{~m}$, por tratarse de un valor estándar para el área de estudio y los radios antes calculados. En esta etapa se eliminaron todos aquellos puntos cercanos con traslapes de visibilidad excesivos, como forma de evitar zonas con sobrevigilancia. Para efectos del análisis, se consideraron sólo aquellos puntos con incidencia directa al interior de los cuadrantes, es decir, cobertura al interior de ellos. Se descontaron, además, todas aquellas zonas catalogadas como primera prioridad correspondientes a asentamientos urbanos.

Selección final de puntos mediante modelo de optimización. Utilizando una modificación del modelo desarrollado por

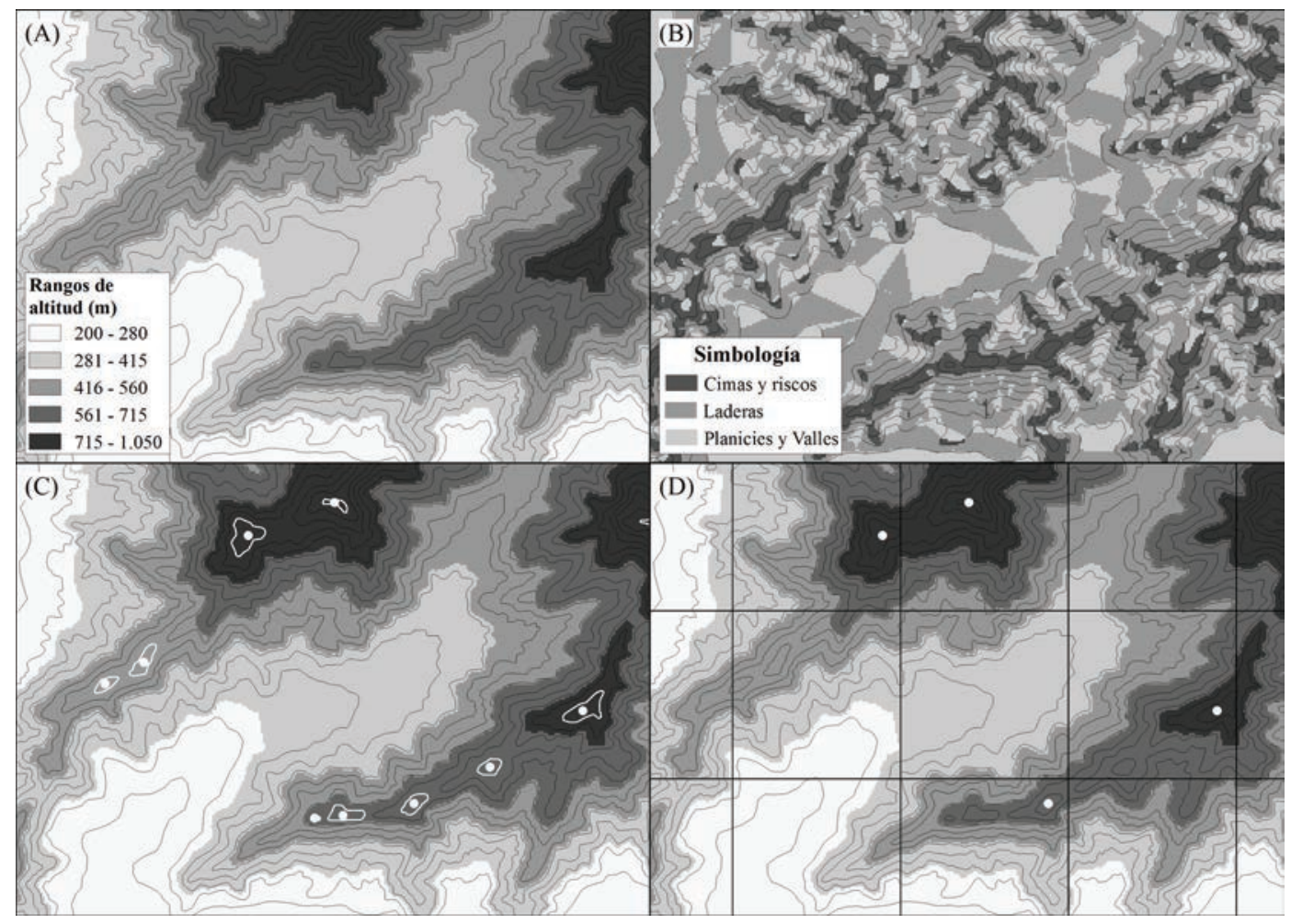

Figura 2. Obtención de puntos opcionales de observación mediante TPI.

Optional observer points obtained with TPI algorithm. 
Echeverría (1995) se analizaron todas las combinaciones de puntos opcionales, buscando obtener la mínima cantidad de ellos capaz de cubrir al menos un $75 \%$ de la superficie de primera prioridad de protección, al interior de cada cuadrante. Para generar las combinaciones requeridas por el análisis, se utilizó un programa de libre distribución desarrollado por García (1999) el cual permite indicar los elementos a combinar y la cantidad de estos a ser seleccionados. Con dicho programa se obtuvo la totalidad de combinaciones requeridas para cada uno de los cuadrantes designados. Luego, a partir de la selección de observadores opcionales, se crearon coberturas vectoriales con los puntos escogidos para cada cuadrante, tomando como límite el archivo raster de prioridades de protección. En la figura 3 se exponen los pasos ejecutados. Cabe destacar que el proceso descrito se iteró para cada una de las combinaciones probadas.

Input por cuadrante

Etapas de procesamiento

Resultados

Puntos candidatos de observación.

Archivo dbf con todas las combinaciones de puntos.

\begin{tabular}{||l|l|l|l|l|}
\hline ID & P1 & P2 & P3 & Priol \\
\hline 001 & A & & & \\
\hline 002 & B & & & \\
\hline 003 & C & & & \\
\hline 004 & A & B & & \\
\hline 005 & A & C & & \\
\hline 006 & B & C & & \\
\hline 007 & A & B & C & \\
\hline
\end{tabular}
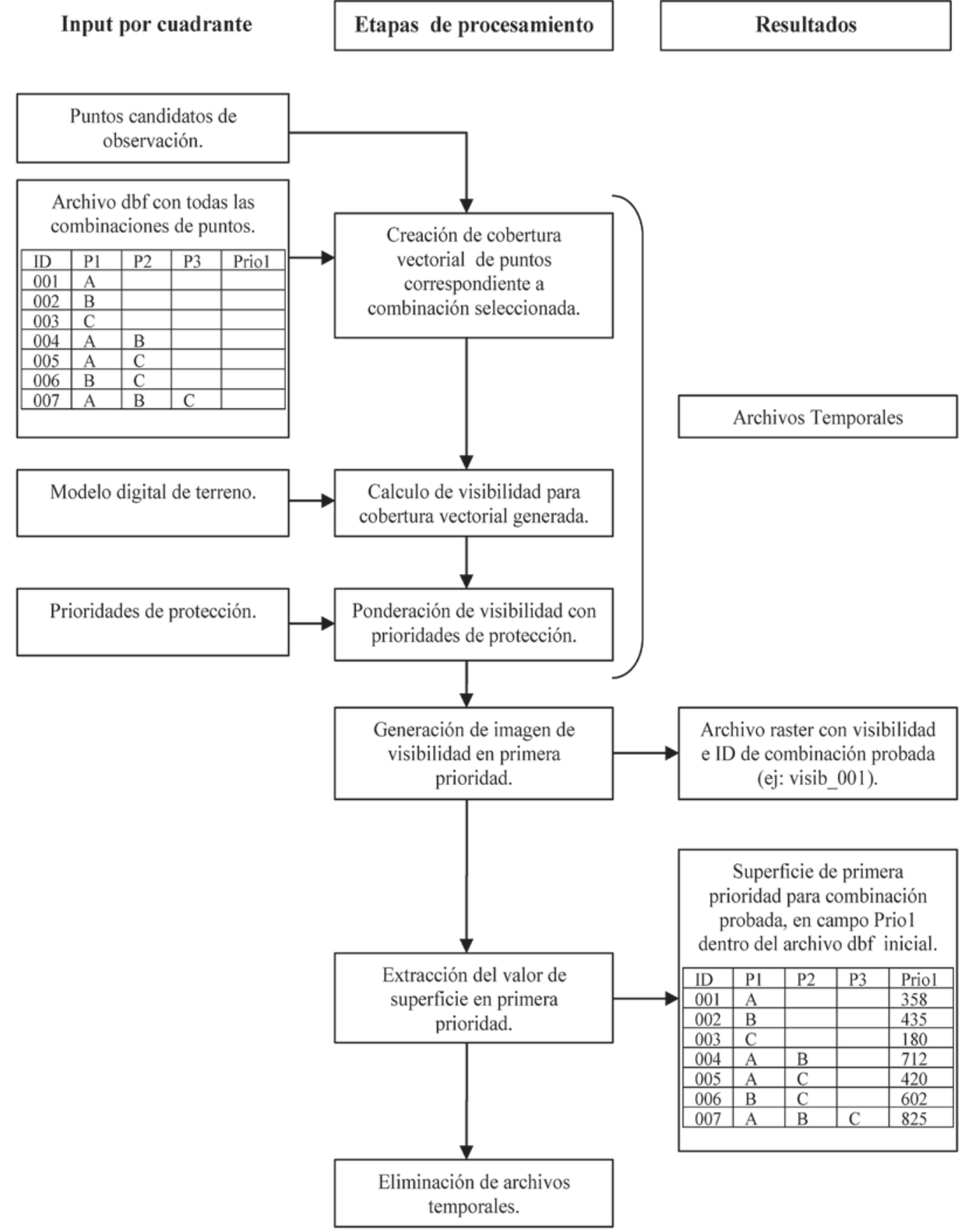

Figura 3. Representación esquemática de código desarrollado para el proceso de datos.

Sketch of phases and code steps for data processing. 
Considerando un tiempo promedio para los análisis de visibilidad de 3 minutos (dependiente del radio de visibilidad además de la extensión y resolución espacial del MDT), la propuesta metodológica desarrollada por Rana y Morley (2002) y utilizada también por Kim et al. (2004) reduce ostensiblemente los tiempos de cómputo, pues de haber considerado un análisis punto a punto para el área de estudio se hubiesen necesitado 31 días para efectos de cálculo. Gracias al método señalado se requirió sólo de 48 horas para realizar los análisis de visibilidad.

La serie de pasos descritos en la metodología para la obtención del conjunto final de puntos candidatos, puede ser comprendida de mejor forma utilizando la figura 4 que contiene un resumen esquemático de las etapas y procedimientos requeridos para la selección final en el área de estudio.

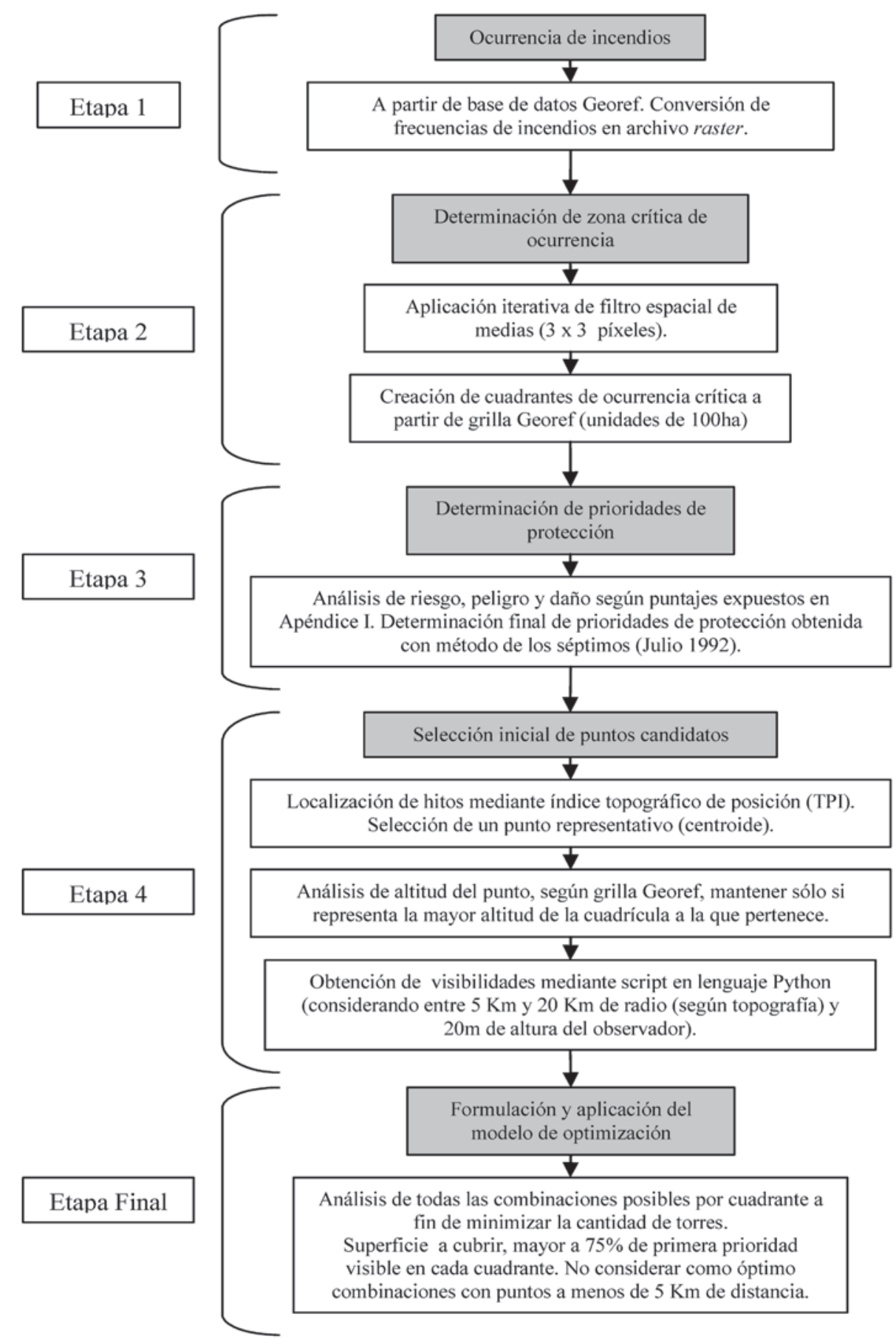

Figura 4. Resumen esquemático de etapas para la selección de torres de detección. 


\section{RESULTADOS}

Delimitación de zonas críticas y cuadrantes de ocurrencia. Mediante el análisis a la cobertura de frecuencia de incendios, se definió un total de 16 zonas críticas al interior de la región Metropolitana. A partir de estas se delimitaron cuatro cuadrantes de ocurrencia. La localización geográfica de las zonas de ocurrencia, así como los detalles de superficie y ubicación de cada cuadrante, se detallan en la figura 5.

El patrón de agrupamiento de la concentración de incendios alrededor de asentamientos urbanos (figura 6) es consistente con las estadísticas de incendios reportadas por CONAF, principalmente, debido a actividades negligentes en el uso del fuego. Los cuadrantes definidos presentan formas irregulares y no se remiten necesariamente al límite de cada zona crítica, por la posibilidad de incluir en los análisis posteriores los territorios adyacentes eventualmente calificados como zonas de alta prioridad de protección. La eficacia del filtro espacial de medias queda en evidencia al observar que los cuadrantes generados contienen el $85 \%$ de los incendios registrados durante el período 2000-2013. Cabe destacar que el cuadrante cuatro, circundante al casco urbano de Santiago, concentra casi un $70 \%$ de estos. A la vez, dentro de este último se localiza la zona de mayor ocurrencia en la región: el Parque Metropolitano de Santiago y sus inmediaciones.

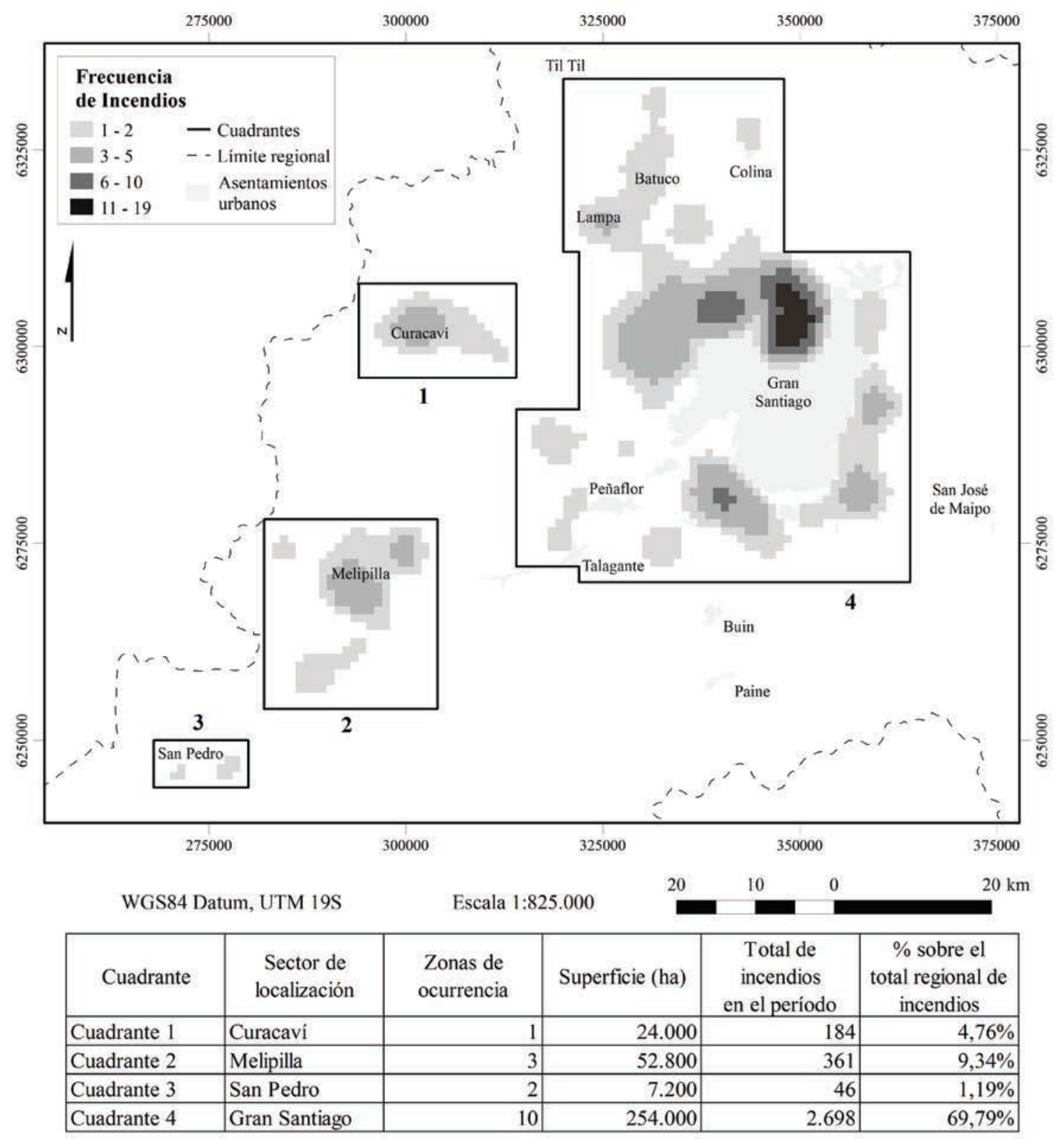

Figura 5. Características y localización de cuadrantes de ocurrencia en la región Metropolitana.

Characteristics and locations of quadrants of fire occurrence in Metropolitan Region. 
Determinación de prioridades de protección. Los cuadrantes delimitados contienen un 53,7 \% de la superficie de primera prioridad al interior de la región Metropolitana. Debido a su localización y extensión, el cuarto cuadrante genera el mayor aporte en superficie de primera prioridad con un $45 \%$. Gran parte de la superficie en primera prioridad tiende a concentrarse en el sector central de la región Metropolitana, contigua al núcleo urbano de la ciudad de Santiago. De igual forma se extienden áreas de primera prioridad hacia la periferia, incluyendo localidades como Tiltil, Colina y Batuco en el norte; San José de Maipo, Pirque y San Bernardo en la zona sur-poniente; además de Curacaví y Melipilla hacia el oriente de la región. La figura 6 muestra la distribución espacial de prioridades de protección con respecto a los cuadrantes de ocurrencia.
Selección de puntos opcionales de observación. A partir del conjunto de procesos descritos en el capítulo de material y métodos y, utilizando como herramienta fundamental el método TPI se identificaron 944 hitos geográficos al interior de la región Metropolitana. Distribuidos heterogéneamente, dichos puntos cubren en su totalidad el área de estudio. La utilización de hitos geográficos del relieve como método para minimizar la cantidad de puntos resulta de gran utilidad en el procesamiento de la información.

Selección final de puntos mediante modelo de optimización. Los cuatro cuadrantes indicados en la figura 5 son analizados ahora desde el punto de vista de la selección de puntos candidato. El cuadrante 1, con una superficie en primera prioridad de protección de 5.856 ha requirió

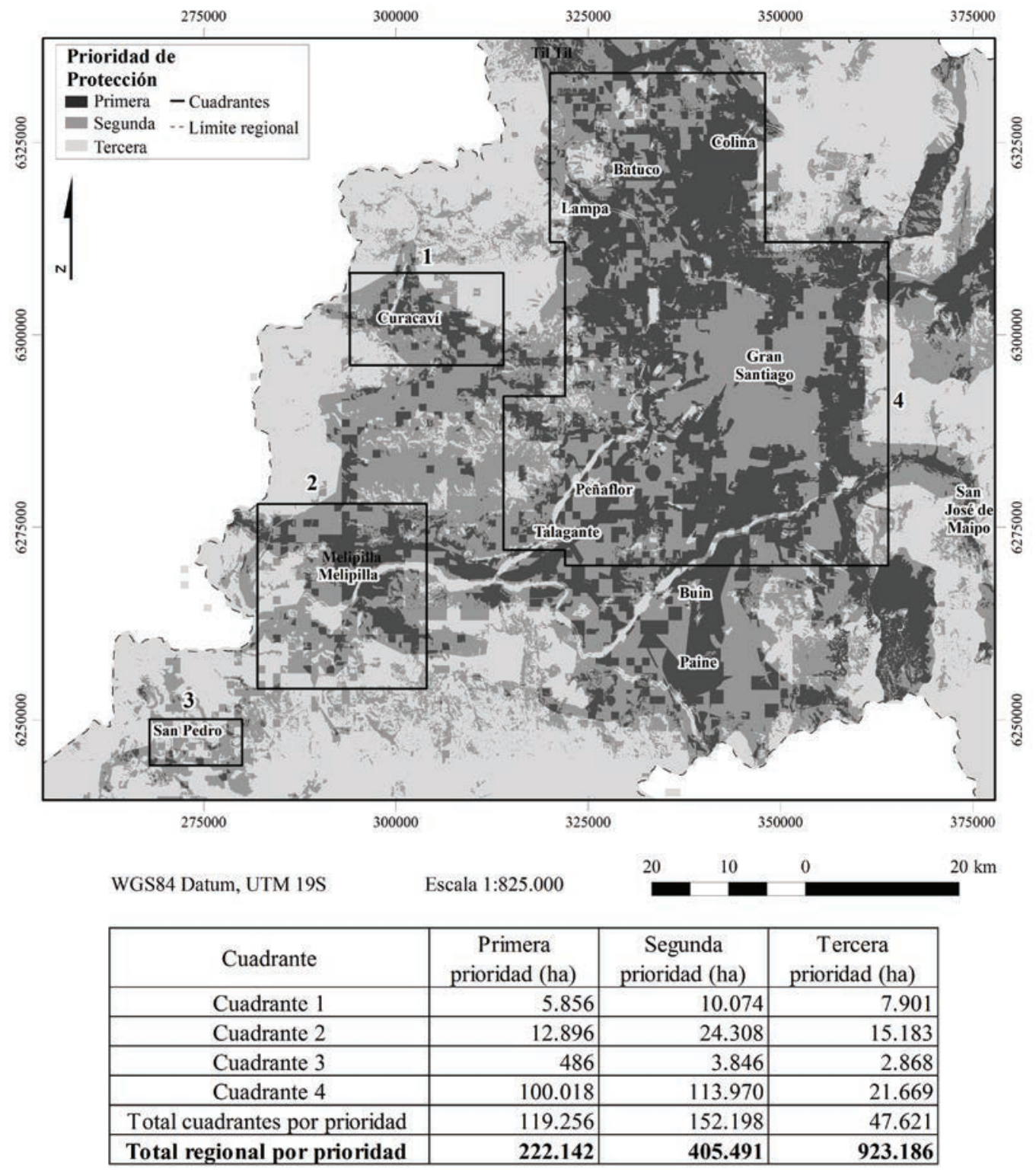

Figura 6. Prioridades de protección para el área de estudio.

Results of analysis priorities for wildfire protection in the study area. 
un mínimo de cobertura de 4.392 ha. Luego de analizar 63 combinaciones posibles, el modelo arrojó como mejor opción los puntos 357 y 373, con un área total visible de 4.907 ha. El detalle para las mejores combinaciones obtenidas según cantidad de puntos se muestra en el cuadro 1.

El punto 373 aparece como opción en cada una de las mejores combinaciones, realizando un aporte de casi un $92 \%$ de la superficie requerida por el modelo. Por otro lado, no existe reemplazo de puntos a medida que aumenta la cantidad de opciones probadas. La incorporación del punto 350 , además de representar un ínfimo aporte en la visibilidad total, no resulta factible por la restricción de distancia impuesta (inferior a $5 \mathrm{~km}$ ). Su inclusión sólo fue admitida para efectos de comparación con el resto de los cuadrantes.

Para el cuadrante 2, se requirió cubrir 9.672 ha de un total de 12.896 ha. Dicha superficie se consiguió utilizando solamente el punto 190, cuya visibilidad en primera prioridad de protección alcanzó las 9.971 ha. Se analizaron de igual forma las 15 combinaciones factibles. Las superficies para las mejores combinaciones obtenidas según cantidad de puntos se muestran en el cuadro 2 .

El punto opcional 190 aporta con un $77 \%$ de la visibilidad en primera prioridad al interior del cuadrante, por lo que corresponde a la mejor opción. Puede observarse el reemplazo del punto 190 en la segunda combinación, esto confirma la condición flexible del modelo al elegir siempre la mejor opción y no estar condicionado a la selección inicial de algún punto.

Con una superficie total de 486 ha en superficie de primera prioridad, en el cuadrante 3 era necesario cubrir al menos 365 ha. Para este caso no hubo necesidad de aplicar el script pues ambos puntos seleccionados cumplían con la condición requerida. El punto 81 obtuvo un total de cobertura de 365 ha, mientras el punto 67 presentó 397 ha cubiertas. Por representar un aporte mayor en visibilidad fue designado este último como opción adecuada para el cuadrante. El requerimiento de cobertura para el cuadrante 4 correspondió a 75.013 ha. Este fue conseguido mediante la combinación de puntos $224,418,435$ y 468 con un total de 76.144 ha en primera prioridad visibles. Por ser el cuadrante más extenso y representativo en el área de estudio, se presentan sus resultados en la figura 7 .

Luego de probar las 1.023 combinaciones posibles los mejores resultados obtenidos según cantidad de puntos se presentan en el cuadro 3.

Queda en evidencia nuevamente la flexibilidad del modelo al apreciar que el punto 418 luego de ser seleccionado en los cuatro primeros niveles de combinaciones, es reemplazado en los niveles cinco, seis y siete, para reaparecer nuevamente en el grupo de ocho elementos.

Propuesta de sistema de torres de detección de incendios para la región Metropolitana. El sistema de torres de detección propuesto está conformado por los ocho puntos, obtenidos como resultado óptimo en los cuadrantes. Dos, en el primer cuadrante (357 y 373 ), uno, para el segundo
Cuadro 1. Superficies visibles en primera prioridad basadas en la mejor combinación de puntos para el cuadrante 1 .

Visible surfaces in first priority based on the best combination of points for quadrant 1 .

\begin{tabular}{lc}
\hline \multicolumn{1}{c}{$\begin{array}{c}\text { Puntos de } \\
\text { observación }\end{array}$} & $\begin{array}{c}\text { Superficie visible en } \\
\text { primera prioridad (ha) }\end{array}$ \\
\hline 373 & 4.029 \\
357373 (solución óptima) & 4.907 \\
357373382 & 5.299 \\
347357373382 & 5.597 \\
347357373382383 & 5.680 \\
347350357373382383 & 5.680 \\
\hline
\end{tabular}

Cuadro 2. Superficies visibles en primera prioridad basadas en la mejor combinación de puntos para el cuadrante 2 .

Visible surfaces in first priority based on the best combination of points for quadrant 2 .

\begin{tabular}{lc}
\hline $\begin{array}{c}\text { Puntos de } \\
\text { observación }\end{array}$ & $\begin{array}{c}\text { Superficie visible en } \\
\text { primera prioridad (ha) }\end{array}$ \\
\hline 190 (solución óptima) & 9.971 \\
131227 & 11.922 \\
131190227 & 12.444 \\
131190206227 & 12.564 \\
\hline
\end{tabular}

Cuadro 3. Superficies visibles en primera prioridad basadas en la mejor combinación de puntos para el cuadrante 4 .

Visible surfaces in first priority based on the best combination of points for quadrant 4 .

\begin{tabular}{lc}
\hline \multicolumn{1}{c}{$\begin{array}{c}\text { Puntos de } \\
\text { observación }\end{array}$} & $\begin{array}{c}\text { Superficie visible en } \\
\text { primera prioridad (ha) }\end{array}$ \\
\hline 418 & 39.034 \\
418435 & 63.034 \\
418435468 & 69.671 \\
224418435468 (solución óptima) & 76.143 \\
224285303435468 & 80.631 \\
224285303435458468 & 84.053 \\
224285303333435458468 & 85.384 \\
224285303333418435458468 & 86.619 \\
224285303324333418435458 & 87.379 \\
468 & \\
224285303324333394418435 & 87.697 \\
458468 &
\end{tabular}




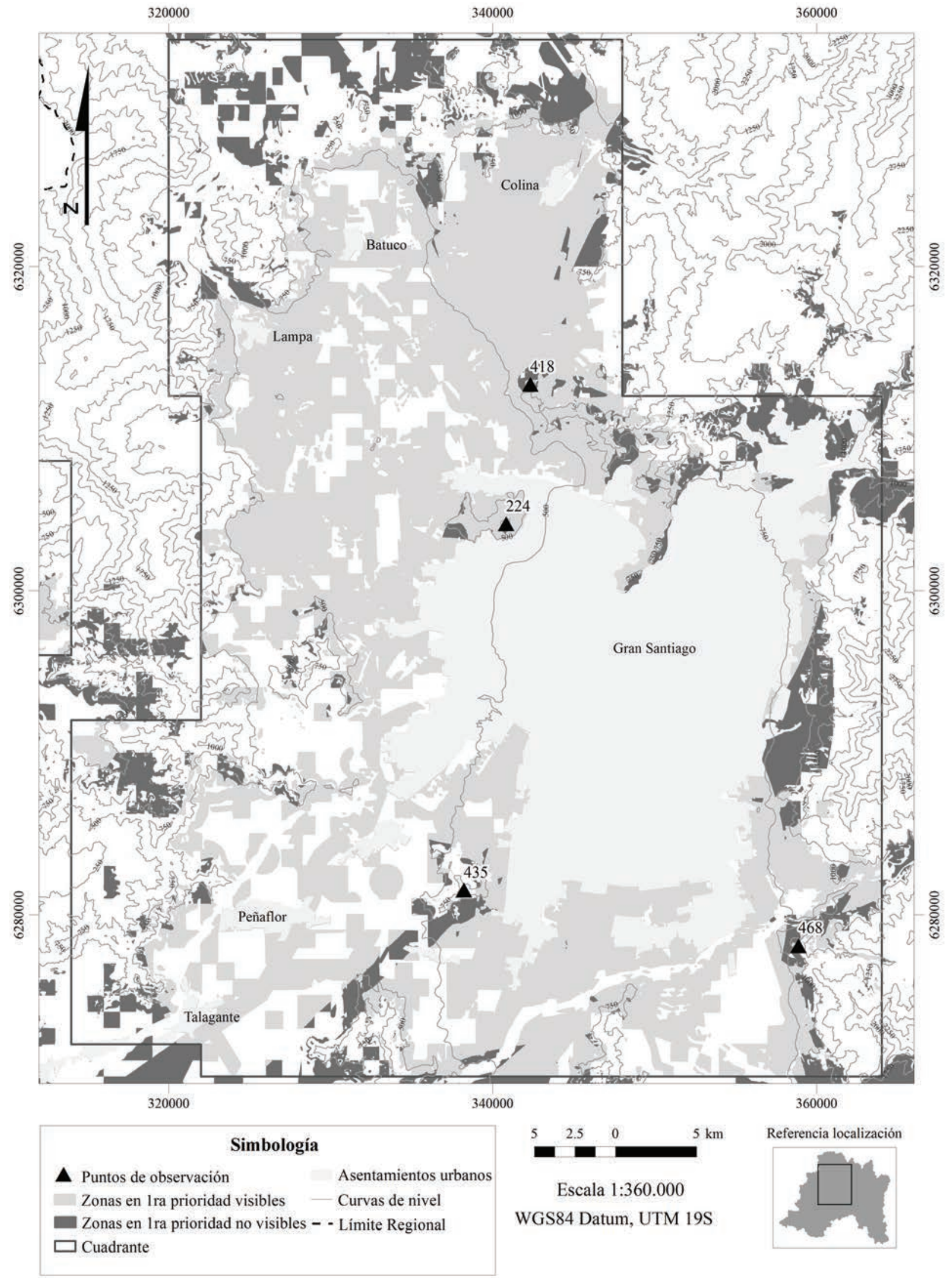

Figura 7. Localización de cuatro posiciones potenciales de observación, en donde se maximiza la primera prioridad de protección. Location of four potential positions of observation, where the first protection priority is maximized. 
y tercer cuadrante (190 y 67, respectivamente), y cuatro, para el cuarto cuadrante $(224,418,435$ y 468). Con una superficie en primera prioridad cubierta de 91.411 ha, el sistema propuesto contiene un 79,3\% de las zonas en igual prioridad al interior de los cuadrantes. A nivel regional son visibles 103.865 ha, equivalentes a un $46,8 \%$ de las áreas en primera prioridad presentes en la región. Las zonas de traslape de visibilidad, útiles para la determinación precisa de focos de incendio, se generan entre todos los puntos del cuarto cuadrante, todos los del tercer cuadrante, y en conjunto para el segundo y primer cuadrante. Finalmente, los radios de visibilidad determinados corresponden a $15 \mathrm{~km}$ para los puntos 190,357 y 373 , y $20 \mathrm{~km}$ para el resto. Los detalles de la localización y zonas de visibilidad para el sistema de torres propuesto se muestran en la figura 8. Las superficies expuestas corresponden al total de visibilidad en primera prioridad para cada punto, sin considerar traslapes, por lo tanto, la sumatoria no equivale al total real visible.

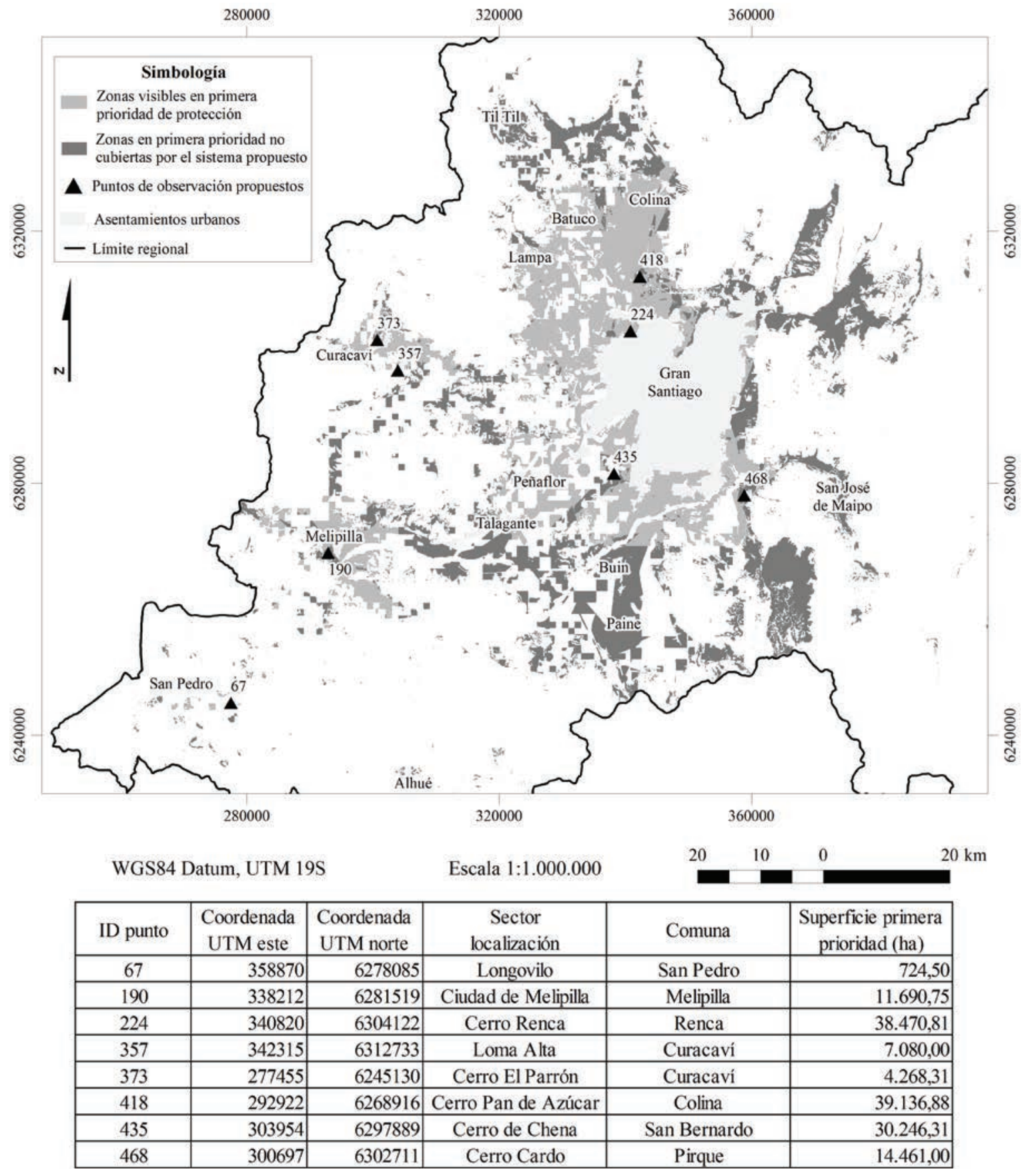

Figura 8. Sistema de torres de detección propuesto para la región Metropolitana.

System detection towers proposed for the Metropolitan Region. 


\section{DISCUSIÓN}

Debido a la influencia de la ocurrencia histórica de incendios, así como la cercanía a centros poblados y caminos a través del componente riesgo, las prioridades de protección presentan cierto nivel de concordancia con las zonas críticas presentadas anteriormente. En contraste, con la cobertura actual en primera prioridad de 24.637 ha proporcionada por las tres torres de detección presentes en la región Metropolitana, cuya superficie visible se ubica principalmente en sectores que colindan con el casco urbano de la ciudad de Santiago, el sistema propuesto incorpora nuevas zonas en lugares donde las quemas agrícolas y forestales representan un real problema (Curacaví y Melipilla, principalmente). Las principales zonas de primera prioridad no cubiertas por el sistema propuesto corresponden a las proximidades de la Ruta 5 Sur entre Paine y el límite con la región del Libertador Bernardo O'Higgins, además del camino que conecta Santiago con la localidad de San José de Maipo; asimismo incluye pequeños sectores correspondientes a aéreas silvestres protegidas como el Santuario de la Naturaleza Yerba Loca y la Reserva Nacional Río Clarillo. Pese a la importancia ambiental que presentan estas últimas, las estadísticas de incendios no resultan significativas de acuerdo al análisis efectuado mediante el filtro espacial de medias en la determinación de los cuadrantes de ocurrencia, por lo que su inclusión no se justificaría bajo esta metodología.

El uso de diferentes longitudes en los radios de visibilidad resulta de utilidad cuando no se dispone de información suficiente para poder restringir la extensión de estos (Rego y Catry 2006, Del Río et al. 2007). Sumado a las características topográficas propias del terreno los radios de visibilidad pueden verse limitados por el índice de refracción atmosférica. Este fenómeno representa la distorsión en las ondas electromagnéticas y haces de luz que atraviesan la atmósfera por sus características de densidad y humedad (Grabner y Kvičera 2006). La refracción atmosférica está incluida como parámetro en las herramientas que incorporan los SIG para análisis de visibilidad, sin embargo, no existen estudios acabados del tema en aspectos relacionados con su efecto sobre la luz, lo que sumado a un proceso complejo de medición, hacen del índice un medio poco factible para restringir los radios en estudios de visibilidad de torres. La contaminación atmosférica también incide negativamente en la visibilidad que pudiesen tener las futuras torres de observación. Tsai (2005) en un estudio a largo plazo realizado en la zona urbana de Tainan (Taiwan) demostró que la visibilidad en dicha ciudad se redujo en casi $10 \mathrm{~km}$ con respecto a las mediciones iniciales realizadas en $1960(20 \mathrm{~km}$ aproximadamente). Las altas concentraciones de material particulado $\left(\mathrm{PM}_{10}\right)$, sumadas a malas condiciones de ventilación repercuten de forma significativa en los niveles de visibilidad. Debido a las condiciones de ventilación y polución presentes en la región Metropolitana, probablemente, exista una incidencia en los radios efectivos de visibilidad.
La utilización de un modelo combinatorio además de permitir explorar todos los resultados posibles, otorga la ventaja de incorporar diversas restricciones según la necesidad de análisis. Desde un enfoque estrictamente topográfico, Márquez (2013) propone un método alternativo basado en una red de intervisibilidad que es construida mediante la combinación de puntos de alta visibilidad y otro conjunto complementario de puntos a observar, dando como resultado una combinación de localizaciones que cubre el área bajo estudio. En esta investigación, y a diferencia de un problema de optimización entero binario las limitaciones de traslape entre componentes son intrínsecamente evaluadas por la combinatoria y no requieren de restricciones individuales. Este aspecto adquiere gran relevancia cuando la cantidad de puntos opcionales a evaluar es numerosa. Por otro lado, la creación del modelo como script para un software SIG, otorga la ventaja de replicar el procedimiento en otros estudios relacionados con cobertura o visibilidad sujetos a restricciones de superficie. Otra de las ventajas es la no dependencia del método en puntos preexistentes por lo que puede ser utilizado tanto para nuevos análisis como para la revisión de sistemas ya establecidos. Sin embargo, el modelo presenta una limitación al momento de abordar la identificación de localizaciones en amplias extensiones de terreno sin variación de altitud, pues la inexistencia de hitos geográficos no permite la calificación de las zonas mediante un índice topográfico e impide la selección de puntos mediante SIG. Otro aspecto que requiere ser evaluado en estudios posteriores, es la conveniencia de incorporar al modelo puntos llanos de baja altitud que podrían tener una razonable visibilidad hacia los cerros y valles. Al respecto, la literatura científica no reporta estudios en donde se considere este criterio de análisis en la topografía. Estudios posteriores deberían evaluar la factibilidad de incluir dichas zonas en los análisis. Se sostiene que al igual que cualquier otra herramienta de ayuda en la toma de decisiones, el procedimiento desarrollado requiere de la corroboración en terreno de los resultados obtenidos por personal calificado y su posterior evaluación, respecto a factibilidad de construcción de acuerdo a las características del terreno, accesibilidad y los costos de instalación y manutención de la estructura. Lo mismo ocurre al establecer un diseño basado en patrullaje terrestre como el realizado por Álvarez (2008) en la región metropolitana, cuyos resultados complementan satisfactoriamente con la cobertura otorgada por la red de localizaciones de torres localizadas en la precordillera de esa región. Este análisis debiera incluir no sólo la distancia a caminos, sino también la pendiente del terreno y la cobertura vegetal, por la necesidad de movilizar al operario si el punto escogido no se encontrase cerca de algún componente de la red vial existente. A modo de ejemplo, Flández (2011) evalúa la cobertura visual a base de cuatro localizaciones de torres en un área de la Región de Los Ríos, pero no considera la altura de la vegetación como criterio de barrera para el cálculo de visibilidad. 
Ciertamente, este último aspecto es de importancia pues en algunas localizaciones puede incidir directamente en el área visible. Ello repercute que en los escasos estudios que reporta la literatura, es necesario incorporar este tipo de aspectos a un modelo, considerando además las restricciones de acceso como variable de decisión y las características de la vegetación y barreras que se presentan en la topografía (Catry et al. 2004). Si bien es cierto no existen estudios científicos referentes a la cuantificación matemática en materia de accesibilidad, se han desarrollado esbozos de metodologías para limitar los análisis en dicho sentido. Mortenson y Miller (2007) realizaron un estudio para calificar las diferentes zonas del parque nacional Lake Clark en Alaska, con el fin de establecer las restricciones de acceso para la colecta de muestras de vegetación. Utilizando una escala de uno a diez, otorgando los máximos valores a aquellas zonas que no permitiesen el ingreso a pie (glaciares, grandes ríos, terrenos privados, etc.), y realizando luego, un análisis conjunto del tipo de vegetación y pendiente; identificaron las áreas inaccesibles del parque.

\section{CONCLUSIONES}

El modelo propuesto permite establecer una propuesta de localizaciones de torres de observación y detección de incendios forestales basada en la combinación espacial óptima de puntos y su interacción matemática entre ellos en cuanto al aporte individual y colectivo en superficie de visibilidad. Los resultados obtenidos consideran adicionalmente la determinación de prioridades de protección contra incendios forestales, lo cual le otorga a la presente investigación una mejora significativa respecto a otros estudios de similares características reportados en la literatura científica.

Mediante la identificación de hitos topográficos y su inclusión gradual al modelo combinatorio, es posible evaluar en forma precisa el aporte individual de cada localización y su relación respecto a las áreas cubiertas en cada categoría en prioridad de protección. La interacción combinatoria permite, además identificar aquellas áreas críticas de ocurrencia de incendios, las cuales son expresadas espacialmente en el sistema de información geográfica.

Como ventaja comparativa, y, a diferencia de estudios anteriores basados en un conjunto de localizaciones preestablecidas el presente estudio, no considera una población inicial de puntos candidatos, sino que obtiene los emplazamientos más adecuados de acuerdo a una serie de criterios topográficos, permitiendo la incorporación de diversas restricciones y su aplicación en diferentes regiones o territorios que presenten variaciones de altitud. En el caso de contar con información en cantidad y calidad suficientes, futuros estudios deberían considerar la accesibilidad como aspecto importante en la selección de puntos opcionales de localización, pues corresponde a un tema relevante en la decisión final de construcción de un puesto de observación.
Indudablemente la potencial realización de un análisis previo a la localización de torres con estas características dependerá de la extensión a evaluar y de la calidad de la cartografía disponible. Para el caso del presente estudio, considerando la disponibilidad solo de cartografía a nivel regional, no resultaría demasiado certera la determinación de zonas accesibles de acuerdo al método antes señalado por la calificación de usos de suelo demasiado gruesa. Cabe recalcar que de existir la información adecuada debe considerarse este punto en futuros estudios, pues independiente de los resultados de visibilidad obtenidos, la decisión final de localización y construcción de torres de observación dependerá de la factibilidad de acceder al lugar escogido, ya sea en vehículo o a pie.

\section{REFERENCIAS}

Aliste R. 2006. Implementación y comparación de dos modelos de optimización para la ubicación de medios aéreos de combate de incendios forestales: (estudio de caso: Afocelca, Portugal). Memoria para optar al Título de Ingeniero Forestal. Santiago, Chile. Facultad de Ciencias Forestales, Universidad de Chile. $61 \mathrm{p}$.

Álvarez G. 2008. Bases para el diseño de un sistema de detección de incendios forestales mediante patrullaje terrestre en un sector de la precordillera de la región metropolitana. Memoria para optar al Título de Ingeniero Forestal. Santiago, Chile. Facultad de Ciencias Agrarias y Forestales, Universidad de Chile. 93 p.

Brown A, Davis K. 1973. Forest Fire. Control and Use. Second Edition. New York, Mac Graw Hill. 686 p.

Castillo M, P Pedernera. 1999. Análisis de la causalidad de incendios forestales en la Quinta Región de Chile. Santiago, Chile. Publicación Departamento de Manejo de Recursos Forestales, Universidad de Chile. 27 p.

Castillo M, G Julio, P Pedernera. 2004. Diseño e implementación de herramientas computacionales para la prevención y combate de incendios forestales. Laboratorio de Incendios Forestales. Departamento de Manejo de Recursos Forestales, Universidad de Chile. 13 p.

Catry F, Almeida R, Rego F. 2004. Produção de cartografia de visibilidades para Portugal Continental. A importância da sua utilização na vigilância contra incêndios florestais. Silva Lusitana 12(2): 227-241.

Chandler C, Cheney P, Thomas P, Trabaud L, Williams D. 1983. Detection. En: Fire in forestry. Forest fire management and organization. New York, USA. John Wiley \& Sons. p. 6973.

CONAF (Corporación Nacional Forestal, CL). 2003. Informe temporada 2002 - 2003. Volumen 1, Regiones III, IV, V y RM. Santiago, Chile. Departamento de Manejo del Fuego, CONAF. 108 p. (Documento de Trabajo No 379).

CONAF (Corporación Nacional Forestal, CL). 2006. Informe estadístico final Unidad de Gestión de Manejo del Fuego. Temporada 2005 - 2006. Santiago, Chile. Departamento de Manejo del Fuego, CONAF. 133 p. (Documento de Trabajo $\left.\mathrm{N}^{\circ} 442\right)$.

CONAF (Corporación Nacional Forestal, CL). 2014. Estadísticas de incendios forestales. Número y superficies afectadas. 
Período 2000-2013. Consultado 2 ene. 2014. Disponible en http://www.conaf.cl/incendios-forestales/incendios-forestales-en-chile/estadisticas-historicas/

Davis K, G Byram, W Krumm. 1959. Forest fire. Control and Use. New York, USA. John Wiley \& Sons. 944 p.

Del Río J, M Mompín, J García. 2007. Aportaciones al cálculo de la distancia de detección de humos de las torres de vigilancia de incendios forestales. GeoFocus (7) 235-255.

Echeverría J. 1995. Modelo de localización de brigadas de combate de incendios forestales mediante patrullaje terrestre. Memoria para optar al Título de Ingeniero Forestal. Valdivia, Chile. Facultad de Ciencias Forestales, Universidad Austral de Chile. 110 p.

Flandez J. 2011. Análisis de cobertura visual de puntos de observación terrestre fija de detección de incendios forestales de CONAF Región de Los Ríos. Trabajo de Titulación para optar al Título de Ingeniero Forestal. Valdivia, Chile. Facultad de Ciencias Forestales y Recursos Naturales, Universidad Austral de Chile. 31 p.

Franklin W, C Vogt. 2004. Multiple observer sitting on terrain with intervisibility on lo-res data. Rensselaer Polytechnic Institute, New York, USA. Consultado 10 ene. 2014. Disponible en http://www.ecse.rpi.edu/Homepages/wrf/pmwiki

García F. 1999. Combinatoria. Cálculo de permutaciones, variaciones y combinaciones con y sin repetición. Consultado 10 ene. 2013. Disponible en http://garciacapitan.99on.com/ programas

Grabner M, V Kvičera. 2006. Refractive index measurements in the lowest troposphere in the Czech Republic. Journal of Atmospheric and Solar-Terrestrial Physics 68(12): 1334-1339.

Julio G. 1992. Método de determinación de Prioridades de Protección. Santiago, Chile. Facultad de Ciencias Agrarias y Forestales, Escuela de Ciencias Forestales. Universidad de Chile. 28 p. (Manual Docente $\mathrm{N}^{\mathrm{o}} 10$ ).

Julio G. 2005. Fundamentos del Manejo del Fuego. Santiago, Chile. Publicación Departamento de Manejo de Recursos Forestales, Facultad de Ciencias Forestales, Universidad de Chile. 325 p.
Kim Y, S Rana, S Wise. 2004. Exploring multiple viewshed analysis using terrain features and optimization techniques. Computers \& Geosciences 30(9-10): 1019-1032.

Lathrop L. 2004. Diseño de un sistema de brigadas terrestres de combate de incendios forestales para la Región Metropolitana. Proyecto de Memoria para optar al Título de Ingeniero Forestal. Santiago, Chile. Facultad de Ciencias Forestales, Universidad de Chile. 38 p.

Marquez J. 2013. Localización óptima de los puntos de mayor visibilidad combinada. Geofocus 13(1): 195-219.

Mortenson D, A Miller. 2007. Path distance analysis to determine accessibility for a long-term vegetation monitoring program Lake Clark National Park and preserve. Alaska Surveying and Mapping Conference 2008, Alaska. Consultado 2 feb. 2014. Disponible en http://irmafiles.nps.gov/ reference/holding/152538.

Pedernera P. 1995. Modelo de optimización para el diseño de sistemas de detección de incendios forestales. Memoria para optar al Título de Ingeniero Forestal. Santiago, Chile. Facultad de Ciencias Agrarias y Forestales, Universidad de Chile. 95 p.

Pedernera P. 2004. Determinación de localización de brigadas de combate de incendios forestales, región del Bio-Bío. Santiago, Chile. Facultad de Ciencias Agrarias y Forestales, Universidad de Chile. 67 p. (Informe Técnico).

Rana S, J Morley. 2002. Optimising Visibility Analyses Using Topographic Features On the terrain. UCL Working Paper Series, Paper 44. 29 p.

Rego A, Catry A. 2006. Modelling the effects of distance on the probability of fire detection from lookouts. International Journal of Wildland Fire 15(2): 197-202.

Tsai Y. 2005. Atmospheric visibility trends in an urban area in Taiwan 1961-2003. Atmospheric Environment 39(30): 5555-5567.

Weiss A. 2001. Topographic position and landforms analysis. Poster presentation, ESRI User Conference, San Diego, California. Consultado 10 dic. 2013. Disponible en http:// www.jennessent.com/arcview/TPI_Weiss_poster.htm.

Recibido: 11.04 .14

Aceptado: 16.09.14 